\section{BMJ Open Respiratory Research}

\title{
Acute exacerbation of idiopathic pulmonary fibrosis: a 10-year single- centre retrospective study
}

\author{
Masatoshi Yamazoe, Hiromi Tomioka
}

To cite: Yamazoe M, Tomioka H. Acute exacerbation of idiopathic pulmonary fibrosis: a 10-year singlecentre retrospective study. BMJ Open Resp Res 2018;5:e000342. doi:10.1136/ bmjresp-2018-000342

- Additional material is published online only. To view please visit the journal online (http://dx.doi.org/10.1136/ bmjresp-2018-000342).

Received 31 July 2018 Accepted 29 August 2018
Check for updates

\section{(C) Author(s) (or their} employer(s)) 2018. Re-use permitted under CC BY-NC. No commercial re-use. See rights and permissions. Published by BMJ.

Department of Respiratory Medicine, Kobe City Medical Center West Hospital, Kobe, Japan

Correspondence to Masatoshi Yamazoe; yamazoe@kcho.jp

\section{ABSTRACT}

Introduction In 2016, an international working group proposed a revised definition and new diagnostic criteria for the acute exacerbation of idiopathic pulmonary fibrosis (AE-IPF). Based on these criteria, AE-IPF was diagnosed regardless of the presence or absence of a known trigger and categorised as triggered (T-AE) or idiopathic (I-AE) AE-IPF. However, the clinical characteristics of the newly defined AE-IPF and clinical differences between T-AE and I-AE are unresolved.

Methods We retrospectively analysed 64 patients with AE-IPF (I-AE (42), T-AE (22)) admitted to our hospital over a 10-year period.

Results I-AE and T-AE cases did not show differences in in-hospital and long-term outcomes (in-hospital mortality: I-AE 52.4\%, T-AE 59.1\%, p=0.61; long-term mortality: $\mathrm{p}=0.68)$. In the $\mathrm{I}-\mathrm{AE}$ group, significantly more patients received corticosteroid therapy before an $\mathrm{AE}$ (I-AE $35.7 \%$, T-AE 4.5\%; $p=0.01$ ). Significantly more patients in the T-AE group had lung cancer (I-AE 7.1\%, T-AE 59.1\%, p<0.001). I-AE occurred more frequently in winter while T-AE did not show seasonality. The white blood cell (WBC) count and haemoglobin $(\mathrm{Hb})$ level were independent predictors of in-hospital deaths in I-AE (WBC: OR 1.87; 95\% Cl 1.09 to 4.95, $p=0.01 ; \mathrm{Hb}$ : OR $0.26,95 \% \mathrm{Cl} 0.04$ to $0.78, p=0.01$ ) but not T-AE.

Discussion With the introduction of new criteria for AEIPF, a retrospective study over a 10-year period showed a lack of prognostic difference between I-AE and T-AE. The WBC count and $\mathrm{Hb}$ level predicted in-hospital outcome in I-AE cases.

\section{INTRODUCTION}

Idiopathic pulmonary fibrosis (IPF) is the most common form of idiopathic interstitial lung disease. IPF usually progresses gradually, with a median survival of 2-4 years; ${ }^{12}$ however, some patients with IPF experience a rapid progression of respiratory failure that has been termed an acute exacerbation of IPF (AE-IPF). AE-IPF was first described in a case report by Kondoh et al in 1993. ${ }^{3}$ The prognosis of AE-IPF is very poor, with a median survival of 3-4 months, ${ }^{45}$ and is one of the main causes of death in patients with IPF. ${ }^{6-8}$

In 2007, the National Institutes of Health-sponsored IPF Clinical Trial Network

\section{Key messages}

Clinical characteristics of acute exacerbation of idiopathic pulmonary fibrosis (AE-IPF) based on the new American Thoracic Society 2016 criteria, and clinical differences between triggered $A E$ (T-AE) and idiopathic $A E(I-A E)$ are unresolved.

- I-AE and T-AE cases did not show differences in in-hospital and long-term outcomes.

- We have clarified clinical characteristics of newly defined AE-IPF.

published international consensus criteria for AE-IPF that required the exclusion of pulmonary infection and other alternative causes for this disease by using such as bronchoalveolar lavage (BAL). ${ }^{9}$ However, in the clinic, it is difficult to determine the presence of alternative causes, in particular pulmonary infection. BAL is also not always useful for the determination of infection and may even worsen the clinical course. Accordingly, the clinical value of BAL is limited in the diagnosis of AE-IPF. ${ }^{1011}$

Definite and suspected AE both show similar outcomes and are indistinguishable. ${ }^{4}$ Moreover, in patients with IPF, $\mathrm{AE}$ and acute respiratory worsening induced by identified causes such as infection have the same effect on patient survival. ${ }^{12}$ For patients with such clinical backgrounds, revised AE-IPF diagnostic criteria have been proposed. ${ }^{13}$ Meanwhile, an international working group proposed a revised definition and new diagnostic criteria for acute AE-IPF in 2016. ${ }^{6}$ Based on these criteria, AE-IPF may be diagnosed regardless of the presence or absence of a known trigger. In addition, AE-IPF can be further categorised as triggered (T-AE) or idiopathic (I-AE), depending on whether an underlying trigger is found. However, the clinical characteristics of newly defined AE-IPF cases and the clinical differences between T-AE and I-AE are not well known. 
We retrospectively analysed newly defined AE-IPF cases admitted to a community teaching hospital over a 10-year period. The aim of this study was to describe the clinical characteristics of such newly defined AE-IPF cases and to identify any clinical differences between T-AE and I-AE.

\section{METHODS}

We retrospectively analysed consecutive patients with AE-IPF admitted to our hospital from January 2008 to December 2017. Using clinical records, AE-IPF was diagnosed based on American Thoracic Society (ATS) 2016 criteria: previous or concurrent diagnosis of IPF; acute worsening or development of dyspnoea of typically $<1$ month duration; CT with new bilateral ground-glass opacity and/or consolidation superimposed on a background pattern consistent with a usual interstitial pneumonia pattern and deterioration not fully explained by cardiac failure or fluid overload. ${ }^{6}$

Pneumonia was defined as the presence of fever or a wet cough, an increased white blood cell (WBC) count or $\mathrm{C}$ reactive protein (CRP) level and the presence of a new focal infiltrate on chest imaging. IPF cases were diagnosed on the basis of 2011 diagnostic criteria for this disease. ${ }^{2}$ All AE-IPF cases were categorised as either I-AE or T-AE depending on whether an underlying trigger was found. Baseline demographic data (age, sex, smoking status, comorbid lung cancer, home oxygen therapy, pulmonary function tests and treatments before $\mathrm{AE}$ ) and laboratory data, vital signs, seasons on admission, treatments after $\mathrm{AE}$ and in-hospital outcomes were obtained from clinical records. The administration of azithromycin was included in antibiotic therapy. However, azithromycin was also analysed separately from other antibacterial drugs since it has been shown to lower AE-IPF patient mortality. ${ }^{14}$ The triggers were divided into the categories of infection, postprocedural/postoperative, drug, aspiration and others. The conditions for a postprocedural $\mathrm{AE}$ were defined as: (1) induced by bronchoscopy or percutaneous procedure and (2) onset within 1 month after the procedure. The conditions for a postoperative $\mathrm{AE}$ were defined as: (1) induced by an operation performed in an operating room and (2) onset within 2 months after the operation. The conditions for a drug-induced $\mathrm{AE}$ were defined as: (1) administration of a causative drug; (2) presence of previous reports about a drug-induced lung injury and (3) onset within 1 month after the last drug administration. We allowed longer periods for the onset of an $\mathrm{AE}$ under the conditions of a postoperative $\mathrm{AE}$ because the operation was more invasive.

Seasons were defined as follows: spring (MarchMay), summer (June-August), autumn (SeptemberNovember) and winter (December-February).

Subsequently, we divided patients with AE-IPF into survivors (patients who were discharged) and non-survivors (patients who died in hospital) and identified predictors of in-hospital deaths. In subgroup analysis, we divided patients with I-AE or T-AE into survivors and non-survivors and identified their respective predictors of in-hospital deaths.

Finally, long-term outcomes in AE-IPF, I-AE and T-AE groups, respectively, were analysed. In cases of patients who required more than one episode of hospitalisation for AE-IPF during the study period, their survival times were calculated from the date of their first hospitalisation.

\section{STATISTICS}

All statistical analyses were performed using JMP software package V.13 (SAS, Cary, North Carolina, USA). Continuous variables were compared using unpaired t-test and Mann-Whitney U test. Categorical variables were compared using $\chi^{2}$ and Fisher's exact tests. Multivariate logistic regression analysis was used to determine independent predictors of in-hospital deaths and to obtain ORs adjusted for possible confounding factors by univariate analysis $(\mathrm{p}<0.05)$. The 95\% CI for each OR was calculated. Statistical significance was determined from the 95\% CI, not including 1.00 for logistic analysis. Survival curves were drawn using Kaplan-Meier estimates and compared with the log-rank test. $\mathrm{P}<0.05$ were considered significant.

\section{RESULTS}

We identified 321 cases with IPF admitted to our hospital during the study period. Of these 321 IPF admissions, 64 cases (57 unique patients) represented AE-IPF. The other cases were admitted for the examination or treatment of lung cancer $(\mathrm{n}=64)$, pneumonia $(\mathrm{n}=56)$, pulmonary rehabilitation $(n=32)$, natural course $(n=30)$, provision of home oxygen therapy $(n=15)$, other infection $(n=14)$, examination for IPF $(n=13)$, treatment for IPF $(n=10)$ or others $(\mathrm{n}=23$; figure 1$)$. The AE-IPF cases consisted of 54 males $(84.4 \%)$ and 10 females $(15.6 \%)$ with a mean age of 76.3 years (range: 60-95 years). Five patients experienced more than one episode of AE-IPF: four patients endured two episodes and one patient four episodes. Of 57 unique patients, nine had not been previously diagnosed with IPF until AE-IPF occurred. For all AE-IPF cases, patient backgrounds, vital signs, laboratory data at $\mathrm{AE}$, treatments after $\mathrm{AE}$ and in-hospital outcomes are shown in table 1. Pulmonary function tests were performed a median of 109 days before AE-IPF. Sixteen comorbid lung cancer cases consisted of six adenocarcinomas, five squamous cell carcinomas, one adenocarcinoma and squamous cell carcinoma, one small cell carcinoma and three histologically unknown. Of the 16, 5 cases were under anticancer therapy: 1 each for carboplatin (CBDCA) and pemetrexed (PEM), CBDCA and paclitaxel, CBDCA and etoposide, PEM and bevacizumab and erlotinib, respectively.

Of the 64 cases, 42 were I-AE cases and 22 were T-AE cases depending on whether a trigger was found. The details of the 22 triggers were follows: 7 postprocedural/ postoperative cases, 6 infection, 5 drug, 1 infection and drug, 1 aspiration and 2 others (figure 1). Of the seven 


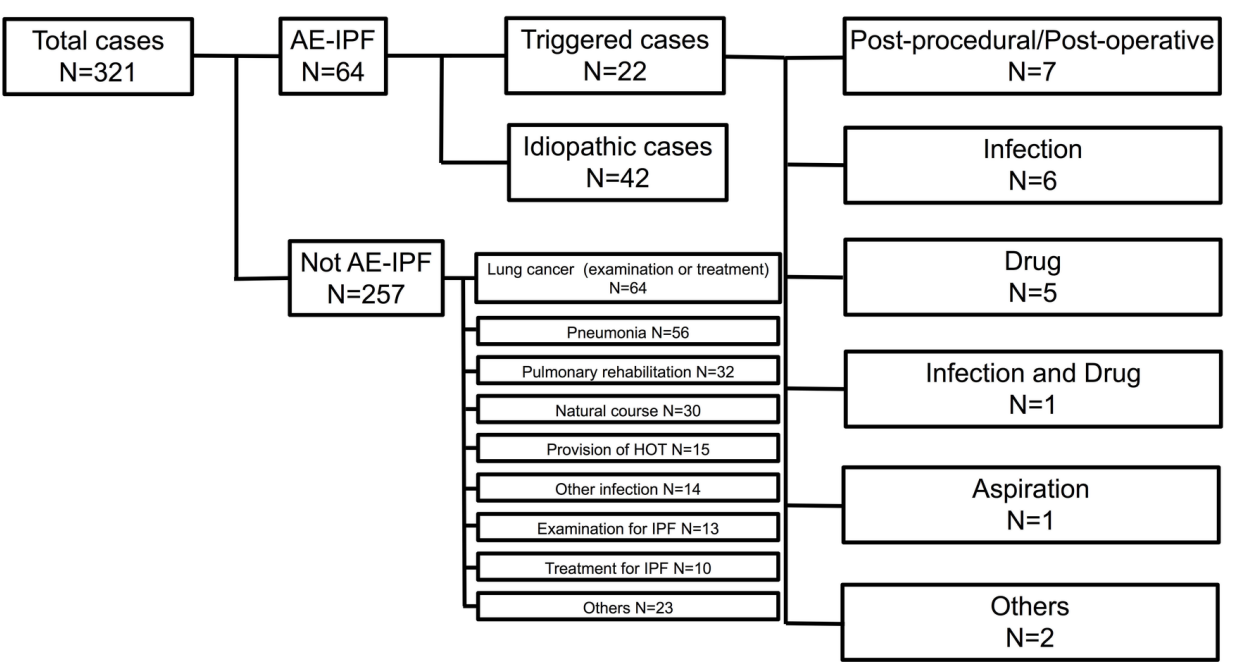

Figure 1 Case selection chart. AE-IPF, acute exacerbation of idiopathic pulmonary fibrosis; HOT, home oxygen therapy; IPF, idiopathic pulmonary fibrosis.

postprocedural/postoperative cases, two were triggered by pulmonary resection for lung cancer, two each by surgical treatment for a pneumothorax or bronchoscopy, respectively and one by an operation for a fracture of the femoral neck. The six infection-induced AE-IPF cases consisted of pneumonia $(n=4)$, cholecystitis $(n=1)$ and enteritis $(n=1)$. Of the five drug-induced cases, four were induced by anticancer drugs (three cytotoxic chemotherapies and one erlotinib) and one by influenza vaccine. In one case, a patient undergoing anticancer therapy developed a lung abscess and an AE occurred soon after. The other two cases were due to an acute myocardial infarction and hepatitis.

Of all AE-IPF cases, 15 occurred in spring, 14 in summer, 15 in autumn and 20 in winter (figure 2). I-AE occurred more frequently in winter: 8 in spring, 8 in summer, 10 autumn and 16 in winter. However, T-AE did not show seasonality: 7 in spring, 6 in summer, 5 in autumn and 4 in winter (figure 2).

Comparisons of the characteristics of I-AE and T-AE groups are shown in table 1 . In the I-AE group, significantly more patients received corticosteroid therapies before $\mathrm{AE}$ and fewer patients had lung cancer than in the T-AE group. The I-AE group showed a significantly lower partial pressure of arterial oxygen $\left(\mathrm{PaO}_{2}\right)$ to inspired oxygen $\left(\mathrm{FiO}_{2}\right)$ ratio $(\mathrm{P} / \mathrm{F})$ at the time of an $\mathrm{AE}$ than the T-AE group $(\mathrm{p}=0.04)$. The two groups displayed a significant difference in smoking status $(\mathrm{p}=0.0048)$. However, the two groups did not show significant differences in age, sex, home oxygen therapy, pulmonary function tests, laboratory data, vital signs, treatments after $\mathrm{AE}$ and in-hospital mortality.

Comparisons of the characteristics of non-survivors and survivors in AE-IPF are shown in table 2. Thirty-five non-survivors showed a median survival time of 24 days (range: 2-119 days). Almost all these patients died due to AE-IPF, except one who died suddenly 7 days after surgery for an intestinal perforation in spite of his stable respiratory status. Non-survivors showed significantly higher WBC counts $(p=0.01)$, CRP levels $(p=0.006)$ and antibiotic therapy rates, and a lower haemoglobin $(\mathrm{Hb})$ level $(p=0.003)$ than survivors. The two groups did not show significant differences in age, sex, home oxygen therapy, pulmonary function tests, laboratory data, vital signs and treatments before AE.

After comparing characteristics between non-survivors and survivors in AE-IPF, WBC, Hb, CRP, antibiotic therapy, age and $\mathrm{P} / \mathrm{F}$ were included in multivariate logistic regression analysis. In general, age is considered a possible confounding factor with various parameters, and the $\mathrm{P} / \mathrm{F}$ ratio has previously been reported as one of the predictors. ${ }^{15-17}$ Consequently, the WBC count (OR 1.38; 95\% CI 1.04-1.83, $\mathrm{p}=0.03$ ) and Hb level (OR 0.51; $95 \%$ CI 0.30 to $0.85, \mathrm{p}=0.01$ ) were identified as independent predictors of in-hospital deaths in AE-IPF (online supplementary table 1 ).

Similarly, we divided patients with I-AE and T-AE, respectively, into survivors and non-survivors and identified their respective predictors of in-hospital deaths (online supplementary tables 2-4). In the I-AE group, non-survivors showed higher WBC counts $(p=0.03)$, blood urea nitrogen (BUN; $p=0.04)$, CRP levels $(p<0.01)$ and antibiotic therapy rates $(\mathrm{p}=0.03)$ and lower $\mathrm{Hb}$ levels $(\mathrm{p}=0.01)$ and $\mathrm{P} / \mathrm{F}(\mathrm{p}=0.02)$ than survivors (online supplementary table 2). WBC, Hb, BUN, CRP and antibiotic therapy were detected as possible confounding factors by univariate analysis; age and $\mathrm{P} / \mathrm{F}$ were included in multivariate logistic regression analysis. Consequently, the WBC count (OR $1.87 ; 95 \%$ CI 1.09 to $4.95 ; \mathrm{p}=0.01$ ) and $\mathrm{Hb}$ level (OR 0.26 ; $95 \%$ CI 0.04 to $0.78 ; \mathrm{p}=0.01$ ) were also identified as independent predictors of in-hospital deaths in I-AE (online supplementary table 3). In contrast, in the T-AE group, a significant difference in the characteristics of non-survivors and survivors was not found (online supplementary table 4); predictors of in-hospital deaths were not identified. 
Table 1 Comparison of patients' characteristics in idiopathic and triggered groups in AE-IPF

\begin{tabular}{|c|c|c|c|c|}
\hline & Total $(n=64)$ & Idiopathic $(\mathrm{n}=42)$ & Triggered $(n=22)$ & $P$ values \\
\hline Gender (Male) & $54(84.4)$ & $34(81.0)$ & 20 (90.9) & 0.47 \\
\hline Age at AE-IPF (years) & $76.3 \pm 6.9$ & $77.0 \pm 6.5$ & $74.9 \pm 7.6$ & 0.25 \\
\hline Smoking status (current/ex/never) & $7 / 53 / 4$ & $1 / 37 / 4$ & $6 / 16 / 0$ & 0.0048 \\
\hline Comorbid lung cancer & $16(25.0)$ & $3(7.1)$ & $13(59.1)$ & $<0.001$ \\
\hline Home oxygen therapy & $26(40.6)$ & $19(45.2)$ & $7(31.8)$ & 0.30 \\
\hline \multicolumn{5}{|l|}{ Pulmonary function tests } \\
\hline VC & $2.0 \pm 0.8(n=35)$ & $2.1 \pm 0.8(n=20)$ & $2.0 \pm 0.8(n=15)$ & 0.66 \\
\hline VC & $62.4 \pm 19.0(n=35)$ & $64.3 \pm 19.1(n=20)$ & $59.8 \pm 19.3(n=15)$ & 0.49 \\
\hline FVC & $2.0 \pm 0.7(n=39)$ & $2.0 \pm 0.6(n=24)$ & $2.0 \pm 0.7(n=15)$ & 0.81 \\
\hline FVC & $64.4 \pm 20.9(n=39)$ & $65.8 \pm 22.8(n=24)$ & $62.2 \pm 17.8(n=15)$ & 0.61 \\
\hline FEV1 & $1.7 \pm 0.6(n=39)$ & $1.7 \pm 0.6(n=24)$ & $1.7 \pm 0.7(n=15)$ & 0.92 \\
\hline \multicolumn{5}{|l|}{ Treatments before AE-IPF } \\
\hline Corticosteroid & $16(25.0)$ & $15(35.7)$ & $1(4.5)$ & 0.01 \\
\hline Immunosuppressive therapy & $2(3.1)$ & $1(2.4)$ & $1(4.5)$ & 1.00 \\
\hline Anti-fibrotic therapy & $8(12.7)$ & $6(14.3)$ & $2(9.1)$ & 0.70 \\
\hline PPI or $\mathrm{H}_{2} \mathrm{~B}$ & $19(29.7)$ & $15(35.7)$ & $4(18.2)$ & 0.16 \\
\hline \multicolumn{5}{|l|}{ Vital signs } \\
\hline $\mathrm{PaO}_{2} / \mathrm{FiO}_{2}$ & $190.0 \pm 125.1$ & $160.0 \pm 73.7(n=28)$ & $236.7 \pm 170.2(n=18)$ & 0.04 \\
\hline Systolic blood pressure $(\mathrm{mm} \mathrm{Hg})$ & $128.0 \pm 23.0$ & $126.2 \pm 21.4$ & $131.5 \pm 25.8$ & 0.38 \\
\hline Pulse (/min) & $98.1 \pm 20.9$ & $96.5 \pm 22.7$ & $101.2 \pm 16.9$ & 0.39 \\
\hline Body temperature $\left({ }^{\circ} \mathrm{C}\right)$ & $37.0 \pm 0.6$ & $37.0 \pm 0.6$ & $36.9 \pm 0.5$ & 0.30 \\
\hline \multicolumn{5}{|l|}{ Laboratory data } \\
\hline WBC $(/ \mu \mathrm{L})$ & $11075.6 \pm 4530.6$ & $10745.0 \pm 4114.9$ & $11706.8 \pm 5280.6$ & 0.42 \\
\hline $\mathrm{Hb}(\mathrm{g} / \mathrm{dL})$ & $12.7 \pm 2.1$ & $12.7 \pm 2.1$ & $12.8 \pm 2.3$ & 0.86 \\
\hline Plt $\left(\times 10^{4} / \mu \mathrm{L}\right)$ & $23.5 \pm 8.6$ & $23.5 \pm 8.5$ & $23.4 \pm 9.2$ & 0.97 \\
\hline $\mathrm{TP}(\mathrm{g} / \mathrm{dL})$ & $7.2 \pm 0.9$ & $7.3 \pm 0.96$ & $7.1 \pm 0.8$ & 0.38 \\
\hline LDH (U/L) & $385.1 \pm 167.0$ & $373.6 \pm 113.9$ & $407.2 \pm 239.9$ & 0.45 \\
\hline BUN (mg/dL) & $19.4 \pm 13.6$ & $18.1 \pm 11.0$ & $21.7 \pm 17.5$ & 0.32 \\
\hline Cre $(\mathrm{mg} / \mathrm{dL})$ & $0.9 \pm 0.6$ & $0.9 \pm 0.7$ & $0.9 \pm 0.5$ & 0.86 \\
\hline $\mathrm{CRP}(\mathrm{mg} / \mathrm{dL})$ & $10.1 \pm 7.8$ & $8.8 \pm 7.0$ & $12.7 \pm 8.8$ & 0.06 \\
\hline $\mathrm{KL}-6(\mathrm{U} / \mathrm{mL})$ & $1425.4 \pm 1004.8(n=58)$ & $1502.5 \pm 993.2(n=40)$ & $1254.1 \pm 1037.8(n=18)$ & 0.39 \\
\hline SP-D (ng/mL) & $374.7 \pm 373.4(n=45)$ & $388.7 \pm 412.5(\mathrm{n}=32)$ & $340.3 \pm 264.7(n=13)$ & 0.70 \\
\hline BAL performed & $4(6.3)$ & $3(7.1)$ & $1(4.5)$ & 1.00 \\
\hline \multicolumn{5}{|l|}{ Treatments after AE-IPF } \\
\hline Intravenous high-dose steroids & $42(65.6)$ & $31(73.8)$ & $11(50)$ & 0.06 \\
\hline Antibiotic therapy & $52(81.3)$ & $32(76.2)$ & $20(90.9)$ & 0.19 \\
\hline Azithromycin & $12(18.8)$ & $9(21.4)$ & $3(13.6)$ & 0.52 \\
\hline Mechanical ventilation & $2(3.1)$ & 0 & $2(9.1)$ & 0.11 \\
\hline $\begin{array}{l}\text { Length of hospital stay, median (range; } \\
\text { days) }\end{array}$ & $27.5(2-119)$ & $26(2-91)$ & $28(3-119)$ & 0.46 \\
\hline In-hospital death & $35(54.7)$ & $22(52.4)$ & $13(59.1)$ & 0.61 \\
\hline
\end{tabular}

Data are presented as a number (\%) or mean \pm SD unless otherwise specified.

AE-IPF, acute exacerbation of idiopathic pulmonary fibrosis; BAL, bronchoalveolar lavage; BUN, blood urea nitrogen; CRP, C reactive protein; Cre, creatinine; FEV1, forced expiratory volume in $1 \mathrm{~s} ; \mathrm{FVC}$, forced vital capacity; $\mathrm{FiO}_{2}$, fraction of inspired oxygen; $\mathrm{H} 2 \mathrm{~B}, \mathrm{H} 2 \mathrm{blocker;} \mathrm{Hb}$, haemoglobin; KL-6, Krebs von den Lungen-6; LDH, lactate dehydrogenase; PPI, proton pump inhibitor; $\mathrm{PaO}_{2}$, partial pressure of arterial oxygen; PIt, platelets. 
(cases)

25

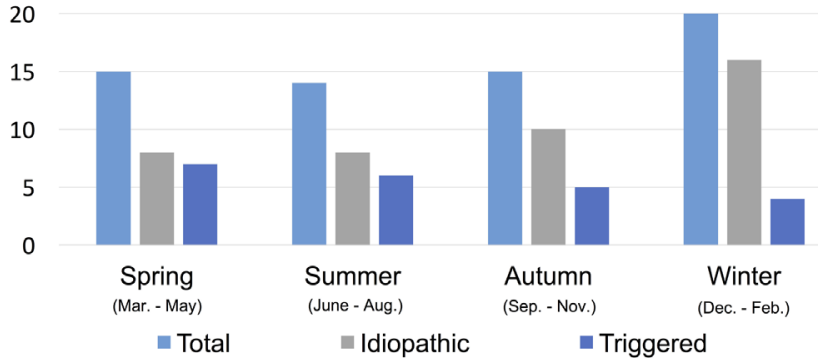

Figure 2 Seasonal variation in the occurrence of AE-IPF. $A E-I P F$, acute exacerbation of idiopathic pulmonary fibrosis.

Finally, Kaplan-Meier survival curves of AE-IPF, I-AE and T-AE are shown in figure 3. A significant difference in overall survival between I-AE and T-AE was not found. With regard to causes of death, 42 patients died of IPF, 3 of lung cancer, 1 of pneumonia and 1 of cholangiocarcinoma.

\section{DISCUSSION}

Some patients with IPF experience AE-IPF, which has a poor prognosis, a median survival of 3-4 months ${ }^{45}$ and is a main cause of death. ${ }^{6-8}$ Definite and suspected AE both show similar outcomes ${ }^{4}$ so that revised AE-IPF diagnostic criteria have been proposed regardless of the presence or absence of a trigger. ${ }^{13}$ However, the clinical characteristics of newly defined AE-IPF cases and the clinical differences between T-AE and I-AE are unclear.

We retrospectively investigated 64 AE-IPF cases, of which 42 were I-AE and 22 were T-AE. The two types of IPF cases, I-AE and T-AE, did not show differences in both in-hospital and long-term outcomes. As for seasonality, I-AE occurred more frequently in winter. In the I-AE group, significantly more patients received corticosteroid therapy before an $\mathrm{AE}$ and fewer patients had lung cancer than in the T-AE group. In addition, the WBC count and $\mathrm{Hb}$ level at the time of an AE were identified as independent predictors of in-hospital deaths in AE-IPF and I-AE.

In this study, triggers were divided into the categories of infection, postprocedural/postoperative, drug, aspiration and others according to ATS guidelines. However, because the guidelines have an unclear definition of each trigger, a level of uncertainty still remains in determining whether infection induces AE-IPF. In this study, we regarded only those $\mathrm{AE}$ cases where infection was certain, as determined by each patient's clinical course and radiological imaging, as infection-induced $\mathrm{AE}$.

Significant differences between I-AE and T-AE groups were not observed for both in-hospital mortality and overall survival. The in-hospital mortality of I-AE (52.4\%) was similar to the mortality of AE-IPF (upward of $50 \%$ and $56.9 \%$, respectively), for which infection was ruled out clinically. ${ }^{515}$ Kishaba et al also conducted a retrospective study using ATS 2016 AE-IPF criteria. ${ }^{16}$ They found that patients with I-AE and T-AE did not show a significant difference in survival, although patients with T-AE tended to have a shorter mean survival (1.4 months vs 11.4 months, $\mathrm{p}=0.094) .{ }^{16}$ This survival result is compatible with our study result in that I-AE and T-AE groups did not show different outcomes.

As previously mentioned, most of the T-AE cases were induced by examinations and therapies for lung cancer; T-AE, therefore, had previously been excluded from AE-IPF. However, our study showed that T-AE did not differ from I-AE in terms of a prognosis. In addition, in a clinical setting, performing BAL in patients with AE-IPF is often difficult because of their severe respiratory condition.

Considering the prognostic similarity between I-AE and T-AE and the wider applicability attributed to the lack of requirement for a BAL, the validity of the newly defined criteria that included T-AE in AE-IPF was highlighted.In the I-AE group, significantly more patients received corticosteroid therapy before the onset of an $\mathrm{AE}$ while fewer patients had lung cancer than in the T-AE group. In the past, corticosteroids and immunosuppressants have been administered to patients with stable IPF. However, in recent years, IPF is thought to be a disease that is associated more closely with abnormal wound healing rather than with inflammation, ${ }^{17}$ for which corticosteroid monotherapy does not provide a clinical benefit. $^{18}$ The 2015 ATS/ERS/JRS/ALAT guidelines recommend avoiding combination therapy with azathioprine, prednisone and $\mathrm{N}$-acetylcysteine for IPF. ${ }^{19}$ In recent years, we have also preferred to avoid corticosteroid use for stable IPF since corticosteroid therapy may be a risk factor for an AE. Atkins et al reported that lower respiratory tract infections occurred more frequently in low-dose prednisolone users and that an AE occurred more frequently in patients receiving immunosuppression therapy, including steroids. ${ }^{20}$ Papiris et al stated that $\mathrm{AE}$ was induced by bacterial infection and steroids and that other immunosuppressants may be risk factors for AE. $^{21}$

In the T-AE group, significantly more patients $(59.1 \%)$ had lung cancer than in the I-AE group $(7.1 \%)$. Examinations, operations and anticancer drug therapies for lung cancer make up most of the AE-IPF triggers. In patients with IPF, developing lung cancer was a serious event in the induction of AE-IPF. Sato et al reported that in lung cancer surgery, an AE occurred in $9.3 \%$ of patients with IPF, who subsequently showed a mortality rate of $43.9 \% .^{22}$ Further, anticancer therapy was also reported to induce an AE, that is, chemotherapy-induced AE of IPF. ${ }^{23} 24$

I-AE occurred more frequently in winter. In previous reports, AE-IPF based on the old diagnostic criteria also occurred more frequently in winter as well as spring. ${ }^{425}$ Although the reason for this seasonality remains unclear, one possible explanation is that an unidentifiable viral infection may play a role in the occurrence of an AE. ${ }^{2627}$ In this study, AE cases that were induced by obvious infection were classified as T-AE. However, cases induced by an 


\begin{tabular}{|c|c|c|c|}
\hline & Survivors $(n=29)$ & Non-survivors $(n=35)$ & $P$ values \\
\hline Gender (Male) & $25(86.2)$ & $29(82.9)$ & 1.00 \\
\hline Age at AE-IPF (years) & $76.6 \pm 5.3$ & $76.1 \pm 8.1$ & 0.78 \\
\hline Smoking status (current/ex/non) & $2 / 26 / 1$ & $5 / 27 / 3$ & 0.42 \\
\hline Comorbid lung cancer & $4(13.8)$ & $12(34.3)$ & 0.08 \\
\hline Home oxygen therapy & $13(44.8)$ & $13(37.1)$ & 0.39 \\
\hline \multicolumn{4}{|l|}{ Pulmonary function test } \\
\hline VC & $2.2 \pm 0.9(n=17)$ & $1.9 \pm 0.7(n=18)$ & 0.44 \\
\hline$\% \mathrm{VC}$ & $66.0 \pm 19.8(n=17)$ & $59.1 \pm 18.1(n=18)$ & 0.29 \\
\hline FVC & $2.0 \pm 0.6(n=19)$ & $2.0 \pm 0.7(n=20)$ & 0.83 \\
\hline FVC & $66.2 \pm 22.6(n=19)$ & $62.7 \pm 19.4(n=20)$ & 0.60 \\
\hline FEV1 & $1.7 \pm 0.7 n=19$ & $1.7 \pm 0.6 n=20$ & 0.94 \\
\hline \multicolumn{4}{|l|}{ Treatments before AE-IPF } \\
\hline Corticosteroid & $7(24.1)$ & $9(25.7)$ & 0.88 \\
\hline Immunosuppressive therapy & $1(3.4)$ & $1(2.9)$ & 1.00 \\
\hline Anti-fibrotic therapy & $3(10.3)$ & $5(14.3)$ & 0.72 \\
\hline $\mathrm{PPI}$ or $\mathrm{H}_{2} \mathrm{~B}$ & $8(27.6)$ & $11(31.4)$ & 0.74 \\
\hline \multicolumn{4}{|l|}{ Vital signs } \\
\hline $\mathrm{PaO}_{2} / \mathrm{FiO}_{2}$ & $220.7 \pm 100.0(n=21)$ & $164.2 \pm 139.5(n=25)$ & 0.13 \\
\hline Systolic blood pressure $(\mathrm{mm} \mathrm{Hg})$ & $132.0 \pm 23.6$ & $124.7 \pm 22.2$ & 0.21 \\
\hline Pulse (/min) & $98.1 \pm 18.7$ & $98.2 \pm 22.8$ & 0.98 \\
\hline Body temperature $\left({ }^{\circ} \mathrm{C}\right)$ & $37.0 \pm 0.6$ & $36.9 \pm 0.6$ & 0.41 \\
\hline \multicolumn{4}{|l|}{ Laboratory data } \\
\hline WBC $(/ \mu \mathrm{L})$ & $9511.7 \pm 3365.0$ & $12371.4 \pm 4989.5$ & 0.01 \\
\hline $\mathrm{Hb}(\mathrm{g} / \mathrm{dL})$ & $13.5 \pm 1.8$ & $12.0 \pm 2.1$ & 0.003 \\
\hline PIt $\left(\times 10^{4} / \mu \mathrm{L}\right)$ & $22.4 \pm 8.7$ & $24.3 \pm 8.6$ & 0.38 \\
\hline $\mathrm{TP}(\mathrm{g} / \mathrm{dL})$ & $7.3 \pm 0.9$ & $7.1 \pm 1.0$ & 0.29 \\
\hline LDH (U/L) & $355.2 \pm 110.0$ & $410.0 \pm 200.8$ & 0.19 \\
\hline BUN (mg/dL) & $16.3 \pm 7.3$ & $22.0 \pm 16.8$ & 0.10 \\
\hline Cre $(\mathrm{mg} / \mathrm{dL})$ & $0.8 \pm 0.3$ & $0.9 \pm 0.8$ & 0.36 \\
\hline CRP (mg/dL) & $7.2 \pm 6.5$ & $12.5 \pm 8.1$ & 0.006 \\
\hline $\mathrm{KL}-6(\mathrm{U} / \mathrm{mL})$ & $1606.6 \pm 123.4(n=26)$ & $1278.2 \pm 786.7(n=32)$ & 0.22 \\
\hline SP-D (ng/mL) & $311.9 \pm 206.6(n=22)$ & $434.8 \pm 480.1(n=23)$ & 0.27 \\
\hline \multicolumn{4}{|l|}{ Treatments after AE-IPF } \\
\hline Intravenous high-dose steroids & $18(62.1)$ & $24(68.6)$ & 0.59 \\
\hline Antibiotic therapy & $20(69.0)$ & $32(91.4)$ & 0.03 \\
\hline Azithromycin & $7(24.1)$ & $5(14.3)$ & 0.35 \\
\hline Length of survival time, median (days) (range) & NE & $24(2-119)$ & NE \\
\hline
\end{tabular}

Data are presented as a number (\%) or mean \pm SD unless otherwise specified.

AE-IPF, acute exacerbation of idiopathic pulmonary fibrosis; BUN, blood urea nitrogen; CRP, C reactive protein; Cre, creatinine; FEV1, forced expiratory volume in $1 \mathrm{~s}$; FVC, forced vital capacity; H2B, H2 blocker; Hb, haemoglobin; KL-6, Krebs von den Lungen-6; LDH, lactate dehydrogenase; NE, not evaluable; PPI, proton pump inhibitor; PaO2, partial pressure of arterial oxygen; PIt, platelets; SP-D, surfactant protein D; TP, total protein; VC, vital capacity; WBC, white blood cell count.

unidentifiable infection may have been included in I-AE. In contrast, T-AE did not show seasonality. One reason is that, as mentioned above, most of the T-AE occurred during examinations or treatments for lung cancer.
The I-AE group showed a significantly lower $\mathrm{P} / \mathrm{F}$ ratio at the time of an AE than the T-AE group. However, causes for the $\mathrm{P} / \mathrm{F}$ ratio difference between the two groups were not determined. 


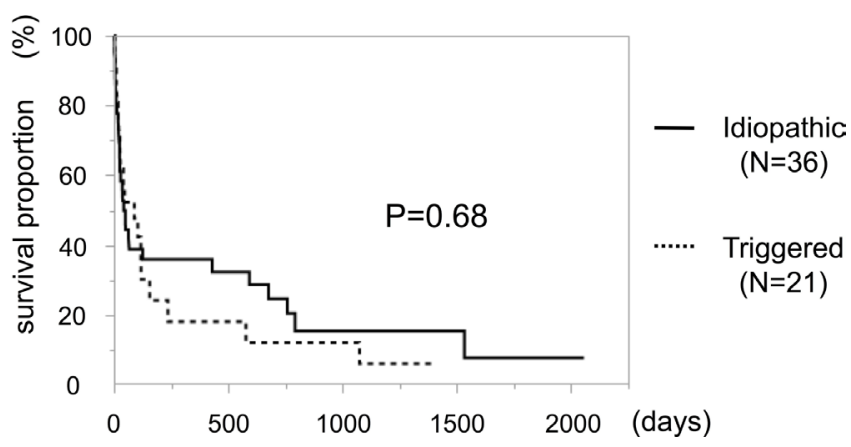

Figure 3 Survival after hospitalisation, idiopathic AE versus triggered $A E$. $A E$, acute exacerbation.

A high WBC count and low $\mathrm{Hb}$ level associated with an $\mathrm{AE}$ were identified as independent predictors of in-hospital deaths both for AE-IPF and I-AE. Although prognostic predictors in newly defined AE-IPF are not well known, several prognostic factors have been identified in AE-IPF in previous studies. Such predictors include CRP, ${ }^{5}$ Krebs von den Lungen-6 (KL-6) ${ }^{15}$ lactate dehydrogenase (LDH) ${ }^{15}$ ${ }^{25} \mathrm{P} / \mathrm{F},{ }^{15}{ }^{28}$ baseline forced vital capacity (FVC) ${ }^{25}$ diffusing capacity of the lung for carbon monoxide, ${ }^{25}$ time between admission and initiation of treatment, ${ }^{25}$ an Acute Physiology and Chronic Health Evaluation II score ${ }^{14}$ and azithromycin therapy. ${ }^{14}$ Song et al suggested that since CRP levels were a prognostic factor of AE-IPF, this implied that inflammation may be a pathogenic mechanism contributing to AE-IPF. ${ }^{5}$ The WBC count is considered to reflect the severity of inflammation of AE-IPF, similar to CRP. Moreover, we identified a low $\mathrm{Hb}$ level as a predictor of in-hospital deaths. However, the reason for this result remains unknown. Kishiba et al identified LDH on admission, the difference in LDH levels between admission and after 2 weeks andthe $\mathrm{P} / \mathrm{F}$ ratio as predictors of 90-day mortality from the onset of AE-IPF based on the new criteria. ${ }^{16}$

This study has several limitations. First, our study is retrospective and therefore subject to the recognised limitations of such a study design, including bias and incomplete data. For example, we had inadequate data on $\mathrm{P} / \mathrm{F}$ and KL-6 values in AE-IPF and pulmonary function results such as FVC. Such variables are predictors of AE-IPF mortality ${ }^{152} 28$ and their deficits may have influenced our study results. However, pulmonary function tests are not often performed before an AE-IPF because IPF is sometimes not diagnosed until AE-IPF occurs. Second, we did not investigate high-resolution CT patterns in AE-IPF. This is important since Akira et al classified newly developing pulmonary opacities into peripheral, multifocal and diffuse patterns and found that survival was worse in patients with a diffuse type than in those with multifocal and peripheral patterns. ${ }^{29}$

\section{CONCLUSION}

We retrospectively analysed data on patients with AE-IPF from a 10-year period, classifying these as either I-AE and T-AE. Differences in both in-hospital and long-term outcomes were not found between I-AE and T-AE. The WBC count and $\mathrm{Hb}$ level were found useful in predicting in-hospital outcome in AE-IPF and I-AE. Newly defined criteria for AE-IPF were also suggested to be valid. However, a prospective validation study is needed to confirm our results concerning newly defined AE-IPF.

Acknowledgements The authors would like to thank the staff at Department of Respiratory Medicine of Kobe City Medical Center West Hospital.

Contributors All authors have directly participated in the planning, execution or analysis of the study. All authors have also read and approved the final manuscript.

Funding The authors have not declared a specific grant for this research from any funding agency in the public, commercial or not-for-profit sectors.

Competing interests None declared.

Patient consent Not required.

Ethics approval Ethics Review Committee of Kobe City Medical Center West Hospital (approval number: 18-001).

Provenance and peer review Not commissioned; externally peer reviewed.

Data sharing statement No additional data are available.

Open access This is an open access article distributed in accordance with the Creative Commons Attribution Non Commercial (CC BY-NC 4.0) license, which permits others to distribute, remix, adapt, build upon this work non-commercially, and license their derivative works on different terms, provided the original work is properly cited, appropriate credit is given, any changes made indicated, and the use is non-commercial. See: http://creativecommons.org/licenses/by-nc/4.0/

\section{REFERENCES}

1. Kim DS, Collard HR, King TE. Classification and natural history of the idiopathic interstitial pneumonias. Proc Am Thorac Soc 2006;3:285-92.

2. Raghu G, Collard HR, Egan JJ. ATS/ERS/JRS/ALAT Committee on Idiopathic Pulmonary Fibrosis. An official ATS/ERS/JRS/ALAT statement: idiopathic pulmonary fibrosis: evidence-based guidelines for diagnosis and management. Am J Respir Crit Care Med 2011;183:788-824.

3. Kondoh Y, Taniguchi H, Kawabata Y, et al. Acute exacerbation in idiopathic pulmonary fibrosis. Analysis of clinical and pathologic findings in three cases. Chest 1993;103:1808-12.

4. Collard HR, Yow E, Richeldi L, et al. Suspected acute exacerbation of idiopathic pulmonary fibrosis as an outcome measure in clinical trials. Respir Res 2013;14:73.

5. Song JW, Hong SB, Lim CM, et al. Acute exacerbation of idiopathic pulmonary fibrosis: incidence, risk factors and outcome. Eur Respir J 2011;37:356-63.

6. Collard HR, Ryerson CJ, Corte TJ, et al. Acute exacerbation of idiopathic pulmonary fibrosis. An international working group report. Am J Respir Crit Care Med 2016;194:265-75.

7. Natsuizaka M, Chiba H, Kuronuma K, et al. Epidemiologic survey of Japanese patients with idiopathic pulmonary fibrosis and investigation of ethnic differences. Am J Respir Crit Care Med 2014;190:773-9.

8. Paterniti $\mathrm{MO}, \mathrm{Bi} \mathrm{Y}$, Rekić $\mathrm{D}$, et al. Acute exacerbation and decline in forced vital capacity are associated with increased mortality in idiopathic pulmonary fibrosis. Ann Am Thorac Soc 2017:14:1395-402

9. Collard HR, Moore BB, Flaherty KR, et al. Idiopathic pulmonary fibrosis clinical research network investigators. Acute exacerbations of idiopathic pulmonary fibrosis. Am J Respir Crit Care Med 2007;176:636-43.

10. Arcadu A, Moua T. Bronchoscopy assessment of acute respiratory failure in interstitial lung disease. Respirology 2017;22:352-9.

11. Petrosyan F, Culver DA, Reddy AJ. Role of bronchoalveolar lavage in the diagnosis of acute exacerbations of idiopathic pulmonary fibrosis: a retrospective study. BMC Pulm Med 2015;15:70.

12. Moua T, Westerly BD, Dulohery MM, et al. Patients with fibrotic interstitial lung disease hospitalized for acute respiratory worsening: a large cohort analysis. Chest 2016;149:1205-14.

13. Ryerson CJ, Cottin V, Brown KK, et al. Acute exacerbation of idiopathic pulmonary fibrosis: shifting the paradigm. Eur Respir $J$ 2015;46:512-20. 
14. Kawamura K, Ichikado K, Yasuda Y, et al. Azithromycin for idiopathic acute exacerbation of idiopathic pulmonary fibrosis: a retrospective single-center study. BMC Pulm Med 2017:17:94.

15. Kishaba T, Tamaki H, Shimaoka $Y$, et al. Staging of acute exacerbation in patients with idiopathic pulmonary fibrosis. Lung 2014;192:141-9.

16. Kishaba T, Nei Y, Momose M, et al. Clinical characteristics based on the new criteria of acute exacerbation in patients with idiopathic pulmonary fibrosis. Eurasian J Med 2018;50:6-10.

17. Gross TJ, Hunninghake GW. Idiopathic pulmonary fibrosis. N Engl $J$ Med 2001;345:517-25.

18. Richeldi L, Davies HR, Ferrara G. Corticosteroids for idiopathic pulmonary fibrosis. Cochrane Database Syst Rev 2003;3:CD002880.

19. Raghu G, Rochwerg B, Zhang Y, et al. An official ATS/ERS/JRS/ALAT clinical practice guideline: treatment of idiopathic pulmonary fibrosis. an update of the 2011 clinical practice guideline. Am J Respir Crit Care Med 2015;192:e3-19.

20. Atkins CP, Loke YK, Wilson AM. Outcomes in idiopathic pulmonary fibrosis: a meta-analysis from placebo controlled trials. Respir Med 2014;108:376-87.

21. Papiris SA, Kagouridis K, Kolilekas L, et al. Survival in Idiopathic pulmonary fibrosis acute exacerbations: the non-steroid approach. BMC Pulm Med 2015;15:162.
22. Sato $\mathrm{T}$, Teramukai $\mathrm{S}$, Kondo $\mathrm{H}$, et al. Impact and predictors of acute exacerbation of interstitial lung diseases after pulmonary resection for lung cancer. J Thorac Cardiovasc Surg 2014;147:1604-11.

23. Kenmotsu H, Naito T, Kimura M, et al. The risk of cytotoxic chemotherapy-related exacerbation of interstitial lung disease with lung cancer. J Thorac Oncol 2011;6:1242-6.

24. Isobe K, Hata Y, Sakamoto S, et al. Clinical characteristics of acute respiratory deterioration in pulmonary fibrosis associated with lung cancer following anti-cancer therapy. Respirology 2010;15:88-92.

25. Simon-Blancal V, Freynet $\mathrm{O}$, Nunes $\mathrm{H}$, et al. Acute exacerbation of idiopathic pulmonary fibrosis: outcome and prognostic factors. Respiration 2012;83:28-35.

26. Huie TJ, Olson AL, Cosgrove GP, et al. A detailed evaluation of acute respiratory decline in patients with fibrotic lung disease: aetiology and outcomes. Respirology 2010;15:909-17.

27. Wootton SC, Kim DS, Kondoh Y, et al. Viral infection in acute exacerbation of idiopathic pulmonary fibrosis. Am J Respir Crit Care Med 2011;183:1698-702.

28. Tomioka H, Sakurai T, Hashimoto K, et al. Acute exacerbation of idiopathic pulmonary fibrosis: role of Chlamydophila pneumoniae infection. Respirology 2007;12:700-6.

29. Akira M, Kozuka T, Yamamoto S, et al. Computed tomography findings in acute exacerbation of idiopathic pulmonary fibrosis. Am J Respir Crit Care Med 2008;178:372-8. 